45 | 2018

Varia

\title{
Le bilinguisme à l'école primaire
}

\section{Franco Calvetti}

\section{(2) OpenEdition}

\section{Journals}

Édition électronique

URL : http://journals.openedition.org/esp/2933

DOI : 10.4000/esp.2933

ISSN : 2532-0319

\section{Éditeur}

Centre d'Information sur l'Éducation Bilingue et Plurilingue

\section{Édition imprimée}

Date de publication : 1 décembre 2018

Pagination : 15-22

ISSN : 1127-266X

\section{Référence électronique}

Franco Calvetti, « Le bilinguisme à l'école primaire », Éducation et sociétés plurilingues [En ligne], 45

2018, mis en ligne le 01 décembre 2020, consulté le 04 décembre 2020. URL : http://

journals.openedition.org/esp/2933; DOI : https://doi.org/10.4000/esp.2933 


\section{LE BILINGUISME PRÉCOCE PAR FRANCO CALVETTI}

\section{Tullio TeLmon}

Parole chiave: bilinguismo, scuola elementaria, Italia, educazione plurilingue Key-words: bilingualism, primary school, Italy, multilingual education

Franco Calvetti, sécrétaire du CIEBP (CMIEB à son époque) pendant plusieurs années, est décédé en décembre 2017; nous lui avons rendu hommage dans cette revue qu'il avait vu naître, sous la signature de Federico Perolto (cf. Éducation et Sociétés Plurilingues $n^{\circ} 44$, juin 2018).

Tout en étant bien conscients de la nécessité d'une actualisation de ce texte, surtout du point de vue bibliographique - il est paru en 1991 - nous pensons qu'il est utile d'attirer l'attention de nos lecteurs sur l'importance de la précoce militance de Franco Calvetti en faveur de l'éducation plurilingue dans l'école italienne. Nous avons donc le plaisir de republier ici un article qu'il avait commis il y a presque trente ans pour la revue Enfance, à une période où il était le responsable didactique d'une école primaire à Turin.

Il en ressort le portrait d'un pédagogue enthousiaste mais réfléchi, qui a su mettre à profit son expérience d'enfant bilingue «à deux langues maternelles», en y introduisant les enseignements de Renzo Titone et de Tullio De Mauro. Des enseignements qui étaient, dans ce temps-là, bien loin d'être universellement accueillis dans les milieux de l'école italienne.

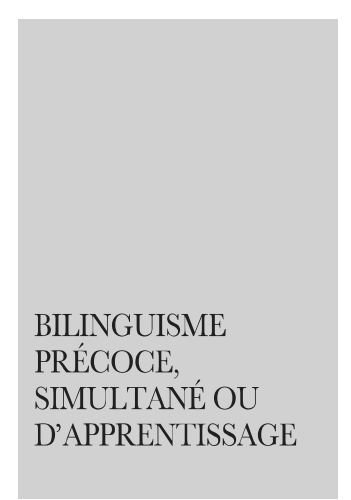

\section{LE BILINGUISME À L'ÉCOLE PRIMAIRE*}

\section{Franco CaLVETti}

$\mathrm{T}$ Très souvent, quand on parle des langues étrangères pratiquées par les enfants, on emploie l'expression «bilinguisme précoce». Cette expression est utilisée en fait pour de nombreuses situations de possession d'une langue étrangère. Il me paraît opportun, pour le sujet que j'aborderai, de faire au moins 


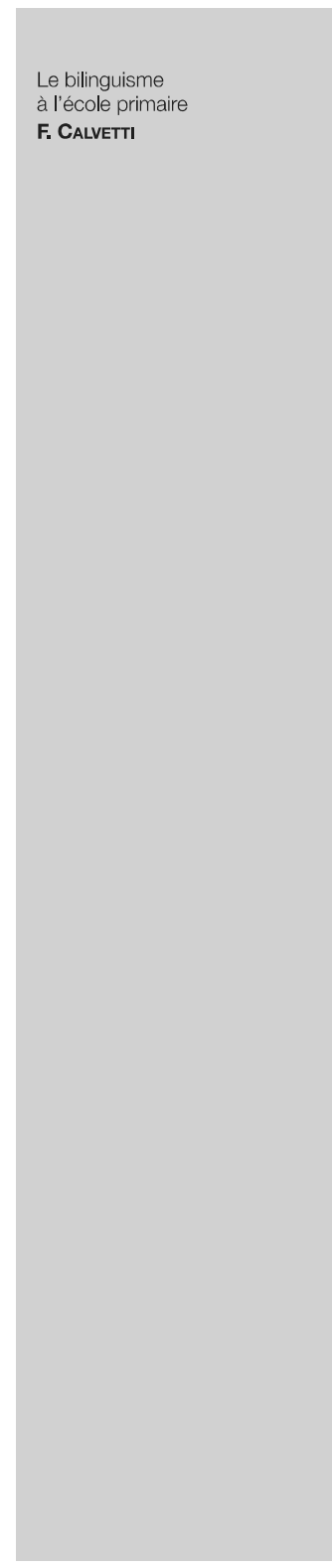

deux distinctions à propos des enfants bilingues précoces

Avec l'expression «bilinguisme simultané», on indique la situation dans laquelle un enfant apprend deux langues simultanément dans les deux premières années de sa vie. Dans ce cas, les deux codes linguistiques sont appris de façon naturelle et directe dans le milieu familial.

C'est souvent le cas de parents de nationalités différentes qui gèrent chacun dans sa propre langue la communication avec leur enfant. Cela fait que l'enfant se trouve en contact avec deux langues qui seront pour lui des langues dites maternelles. Le phénomène du bilinguisme simultané peut se rencontrer aussi dans le cas où des parents parlant la même langue confient leur enfant à une nurse qui s'exprime uniquement et toujours dans sa première langue.

On peut parler de bilinguisme simultané si l'enfant parlant une langue dans sa famille se trouve en contact continu avec une autre langue propre à la communauté dans laquelle il vit.

Avec l'expression «bilinguisme d'apprentissage», on indique le cas des enfants qui, n'ayant pas la possibilité d'apprendre une deuxième langue ni dans la famille ni grâce à leur nurse ni directement dans le milieu social, apprennent la langue étrangère consécutivement à leur langue maternelle, à l'âge préscolaire ou scolaire. Il s'agit toujours de bilinguisme précoce car le bas âge auquel la langue est pratiquée justifie la notion de précocité.

La motivation à l'apprentissage d'une deuxième langue dans le contexte scolaire est généralement plus faible que dans le cas d'apprentissage simultané en famille ou dans le milieu plus proche où l'enfant est appelé à s'exprimer pour des questions de nécessité, pour satisfaire ses besoins primaires.

Dans le contexte scolaire, la motivation à l'apprentissage doit être toujours stimulée et induite artificiellement. Il faut dire que la motivation ainsi que l'intérêt, dans le contexte scolaire, sont plus forts chez les enfants de migrants car le besoin d'apprendre la langue du pays d'accueil est ressenti de manière plus prenante.

Dans le cas du bilinguisme d'apprentissage, l'enfant ne passe pas à travers les stades de développement caractéristiques de l'acquisition de la langue maternelle. L'apprentissage est acquis de manière plus consciente.

L'acquisition s'appuie sur la langue maternelle (Kenyeres 1938). Cette définition de cas (enfant exposé à un bilinguisme en famille ou en milieu communautaire et enfant exposé au bilinguisme à l'école postérieurement à la possession de la langue maternelle) nous amène à parler de: 


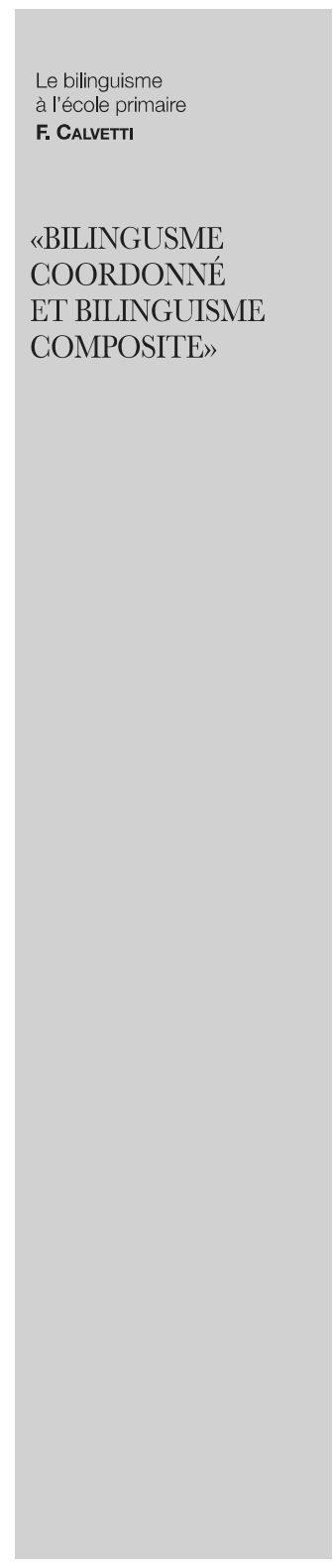

Nous dirons tout de suite que même un enfant qui apprend deux langues simultanément n'arrivera jamais à un degré de bilinguisme tel qu'on pourrait parler d'équilinguisme (Balkan 1970). Il y aura toujours une des deux langues dominante dans les différentes stratifications de la possession langagière.

Mais nous verrons que chez l'enfant apprenant simultanément deux langues, et deux comportements langagiers, le bilinguisme sera du type coordonné, c'est-à-dire que l'enfant montrera une habileté à penser directement dans les deux langues (Osgood et Sebeok 1965) et le lien qui s'établira entre sa pensée et son expression sera évident. Le bilinguisme auquel on se réfère dans cet exposé est donc, de nature consécutive, de type composite, acquis en situation d'apprentissage. Pourquoi apprendre une langue en âge préscolaire et scolaire et précisément en maternelle et en classe d'école primaire?

Nous avons des motivations, ou plus simplement des raisons, pour cette approche à un âge que l'on aime qualifier de précoce.

Des motivations neurophysiologiques ont mis en évidence la toute particulière plasticité que le cerveau des enfants présente avant l'âge de dix ans.

Nous nous référons aux études de Lenneberg (1967) qui a observé que l'acquisition de la première langue est réduite à l'époque de la puberté chez les enfants souffrant d'aphasie. Déjà Penfield (1959) avait noté que les enfants aphasiques étaient en mesure de reconquérir l'usage complet du langage à la différence des adultes pour lesquels cela s'avérait impossible.

Des raisons de caractère psychologique nous apprennent que l'enfant en bas âge est moins inhibé et moins assujetti à se faire conditionner par la peur de se tromper. En outre, il présente davantage de réceptivité à l'apprentissage.

Cette raison a été mise en évidence et étudiée par Titone (1972) et Schumann (1975) l'a confirmée. Krashen (1981) nous parle des grandes possibilités de répétition phonologique chez l'enfant et des difficultés que les adultes présentent à décoder son et rythme d'une langue étrangère.

Les raisons de caractère formatif nous font dire que l'étude de la deuxième langue a un effet considérable pour un développement plus harmonieux de l'enfant. Des recherches (Albert et Obier 1978) nous donnent, grâce à un examen nécropsique du cerveau de personnes polyglottes, l'assurance que le cerveau des bilingues est plus développé car les circonvolutions cérébrales sont plus développées que chez ceux qui parlent une seule langue. Du point de vue plus strictement pédagogique, nous devons avouer qu'en 


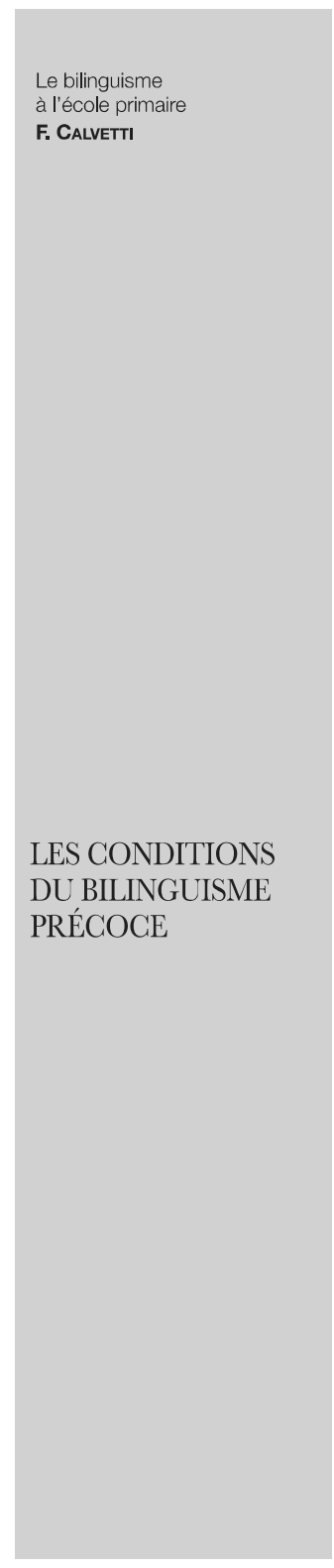

offrant un nouveau modèle d'organisation des connaissances, l'étude et la pratique d'une langue étrangère renforce la «plasticité intellectuelle» (Balkan 1970) et influe de manière considérable sur le développement de la personnalité.

Nous devons effectivement reconnaître que savoir comprendre et utiliser une langue autre que la langue maternelle veut dire élargir les stratégies linguistiques chez l'enfant et étoffer sa conscience métalinguistique. C'est pour cela que nous sommes convaincus que l'apprentissage d'une langue étrangère accroît la capacité de communication en général et en conséquence aussi le maniement de la langue maternelle.

Les raisons du point de vue culturel nous font dire que la confrontation avec d'autres réalités culturelles favorise chez l'enfant la réduction de l'ethnocentrisme et développe son sens de la tolérance.

Ainsi sa capacité à comprendre les expériences et les opinions différentes des siennes l'aidera à manifester son respect pour les autres. Titone (1980) nous dit que «l'apprentissage des langues influence radicalement l'ouverture mentale, l'expansion affective et sociale de l'individu humain». Ce qui revient à dire qu'en plus du développement d'une dimension linguistique nous travaillons aussi dans une dimension éthique.

II y a trois autres questions à aborder si on veut amorcer le problème du bilinguisme à l'école: d'abord «à quel âge commencer?», ensuite «quelle langue enseigner?» et il ne faut pas oublier non plus «qui enseignera la langue étrangère?».

À quel âge? Un auteur comme Titone (1983) est favorable à l'idée de commencer l'apprentissage d'une deuxième langue à partir de quatre-cinq ans.

«La première langue paraît se stabiliser à l'âge de quatre-cinq ans et on peut conseiller que l'âge de quatre ans soit retenu comme le meilleur.» II y a des chercheurs qui pensent qu'il faut faire attention à l'interférence des deux langues qui serait due à la difficulté d'arriver en bas âge à la structuration et à la stabilisation de la première langue. En Italie d'ailleurs, nous avons relevé (enquête doxa di 1984) qu'environ $47 \%$ des familles parlent le dialecte et qu'ainsi la langue étrangère devrait s'ajouter à deux codes linguistiques en train de s'affirmer. Selon Titone, ces craintes ne sont pas justifiées: «II est recommandé que l'enfant s'empare de deux ou plusieurs langues comme si elles faisaient partie simultanément et de manière intégrante d'un système multiple d'expression.» II dit quand même que cet apprentissage à un âge précoce doit se 


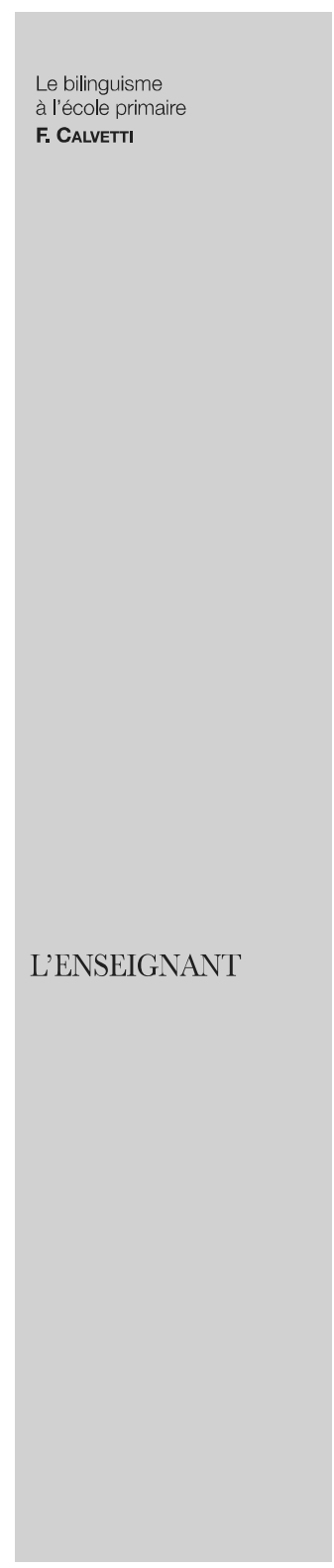

faire avec des temps et des stratégies méthodologiques adaptées. Toujours Titone (1982) stigmatise ceux qui parlent d'identité nationale à préserver par l'apprentissage d'une seule langue chez les enfants. «Le concept d'identité culturelle introduit par la philosophie raciste, repris par les courants sociologiques de la psychanalyse apparaît comme le plus dangereux et comme celui qui provoque des dégâts de formation. Le concept d'identité culturelle donne à l'individu l'idée d'appartenir à un clan mais le sépare des autres individus et groupes qui vivent dans la même société ou dans des sociétés proches ou lointaines. Ce concept accentue la mentalité de défense qui se traduit souvent par des comportements agressifs ou de refus.»

Titone et avec lui beaucoup d'autres sont là pour nous dire que chercher à rapprocher l'enfant de personnes qui parlent une autre langue et se réfèrent à une autre civilisation veut dire aussi travailler pour le dialogue, l'interculturalisme et la paix.

La deuxième question qu'on se pose au moment où on établit qu'une langue peut entrer dans le curriculum de l'enfant de l'école enfantine ou de l'école primaire concerne le choix de la langue à enseigner.

Il y a eu un ample débat en Italie où les programmes ministériels de l'école primaire semblent privilégier l'anglais. Les psychologues nous disent que l'enfant ne présente pas une prédisposition à une langue particulière. Le choix revient donc en premier lieu à la structure scolaire, compte tenu de l'opinion des parents.

II faut aussi aborder le problème de savoir qui devra enseigner à l'école maternelle ou à l'école primaire la langue étrangère. L'acquisition de la langue étrangère peut se faire grâce à un enseignant natif du pays de la langue d'enseignement, grâce à l'instituteur(trice) qui connaît une langue étrangère ou bien grâce à un(e) spécialiste qui intervient de façon extérieure à l'école.

Nous avons examiné beaucoup d'expériences d'apprentissage de langue étrangère dans les différents pays et nous avons observé que souvent l'insuccès de l'apprentissage est strictement dû à l'enseignant.

L'enseignant doit connaître de manière adéquate la langue qu'il doit enseigner et doit connaître de façon sérieuse la didactique de cette langue: une importante formation linguistique et une remarquable habileté méthodologique sont donc demandées. Nous ne croyons pas qu'un étranger parlant sa langue puisse avoir plus de succès auprès des enfants que l'enseignant qui a appris la langue sur laquelle s'appuie l'apprentissage des enfants. Nous avons sou- 


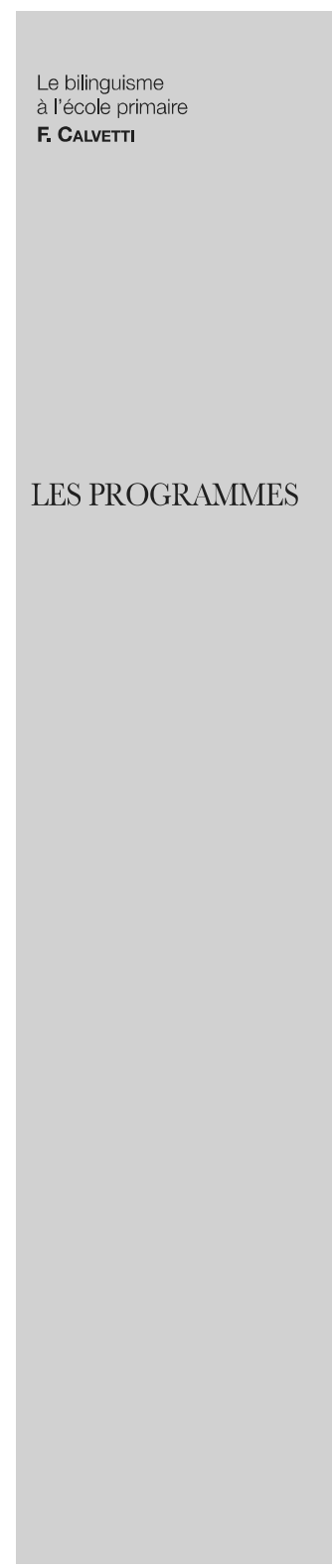

vent remarqué qu'une très bonne préparation didactique amène même à des résultats très positifs pour des personnes ayant quelques défaillances dans la langue qu'ils enseignent.

Le vrai problème est de pouvoir faire référence à des cours rigoureux et de qualité pour tous les enseignants qui font preuve d'attention et d'intérêt pour cette activité. En attendant que la formation préalable au recrutement puisse se faire, il faut mettre sur pied des stages de formation pour les enseignants en service. Nous sommes convaincus que les enseignants du secondaire ne sont pas, vu leur ignorance de la psychopédagogie, les plus indiqués pour s'atteler à cette tâche, délicate et pleine de responsabilité aussi du point de vue affectif.

Plusieurs pays européens, pour s'en tenir à l'Europe, ont pris en charge par le moyen de leurs structures préscolaires et scolaires des classes primaires l'enseignement d'une langue étrangère. Pour certains pays il s'agit de projets, pour d'autres d'expérimentations, pour d'autres de réalisations suivies. L'état des recherches sur le bilinguisme, la demande sociale des parents d'élèves, la réorganisation des projets éducatifs de la part des enseignants amènent à s'occuper de plus en plus du bilinguisme que nous avons appelé précoce.

L'échéance de 1992-1993 accélère ce processus de mise en place de projets et de plans de travail.

En Italie, les programmes ministériels de 1985 actuellement en vigueur pour les trois premières années de l'école primaire portent l'attention des enseignants sur l'enseignement de la langue étrangère dans les cours primaires.

Un projet expérimental de grande importance scientifique pour l'évaluation des résultats a été élaboré, organisé et porté à terme, à la fin de cette phase d'expérimentation, par le ministère de l'Instruction publique de 1978 à 1983.

Examinons brièvement le contenu de ce chapitre dans le texte officiel des Programmes.

La langue étrangère fait partie de l'éducation linguistique en général et se pose trois objectifs:

- aider et enrichir le développement cognitif, en offrant un autre instrument d'organisation des connaissances;

- permettre à l'enfant de communiquer avec les autres à travers une langue différente de la sienne;

— à travers l'instrument linguistique, amener l'élève à la compréhension des autres cultures et des autres peuples.

Le texte est assez ambigu sur le choix de la langue: il est dit que le 


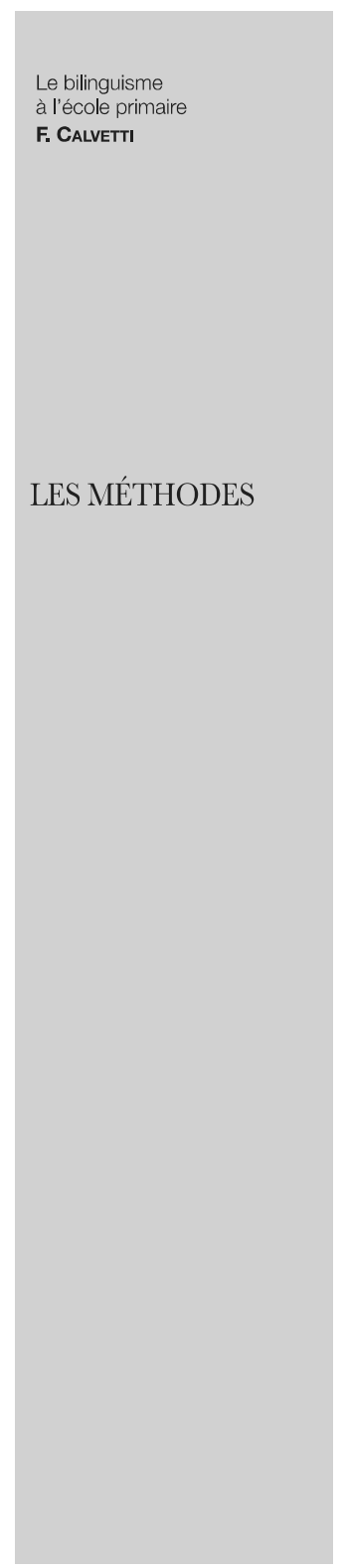

caractère véhiculaire de la langue anglaise offre des occasions plus fréquentes d'expériences soit pour ce qui concerne les moyens de communication, soit pour les échanges internationaux.

Dans le programme, l'âge où commencer l'enseignement de la langue n'est pas fixé: on parle en termes généraux de classes sans indiquer le début.

Une loi d'application, qui tarde à arriver, donnera des indications plus précises de réalisation (classe de début, durée horaire, statut de l'enseignant). Il est dit qu'à la fin du cours primaire l'enfant devra être en mesure de soutenir une conversation facile et savoir lire un petit texte qui se rapporte aux expériences concrètes de la vic quotidienne.

Pour arriver à ce but cognitif et de comportement linguistique, le choix d'une méthode ou mieux de stratégies méthodologiques revêt une grande importance.

Les stratégies méthodologiques, en résumé, doivent tenir compte - de la séquence compréhension-assimilation-production;

- du fait que l'apprentissage donne la priorité à l'oral (compréhension et réponse);

— du fait que le passage à l'écrit (lecture et production) aura lieu seulement à un stade ultérieur;

- de l'opportunité de développer une communication fonctionnelle, ayant largement recours au contexte situationnel;

- du fait que l'apprentissage se fera sous une forme ludique où la motricité et le chant entrent en jeu avec la parole;

- de l'abandon de l'étude de la grammaire traditionnelle à la faveur des réflexions linguistiques et de déductions à partir de situation de contrastes et d'analogie entre l'italien et la L 2;

- du recours aux moyens audiovisuels pour varier les formes d'apprentissage;

- de la correspondance interscolaire pour offrir aux enfants des occasions concrètes de pratique de la langue.

Il s'agit pour l'Italie et pour son école primaire ainsi que pour beaucoup de pays européens de «tâtonnements» à la mode de Freinet: on va à la recherche d'un projet-action, avec la mise au point des problèmes, la mise en valeur des recherches psycholinguistiques et de la didactique des langues, la prise en compte des différentes situations sociales.

Je crois que ce projet-action d'apprentissage d'une langue autre que la sienne en âge scolaire ou même préscolaire peut nous apporter beaucoup sur bien des plans: sur le plan cognitif, bien sûr, comme occasion de réorganisation des connaissances autour 


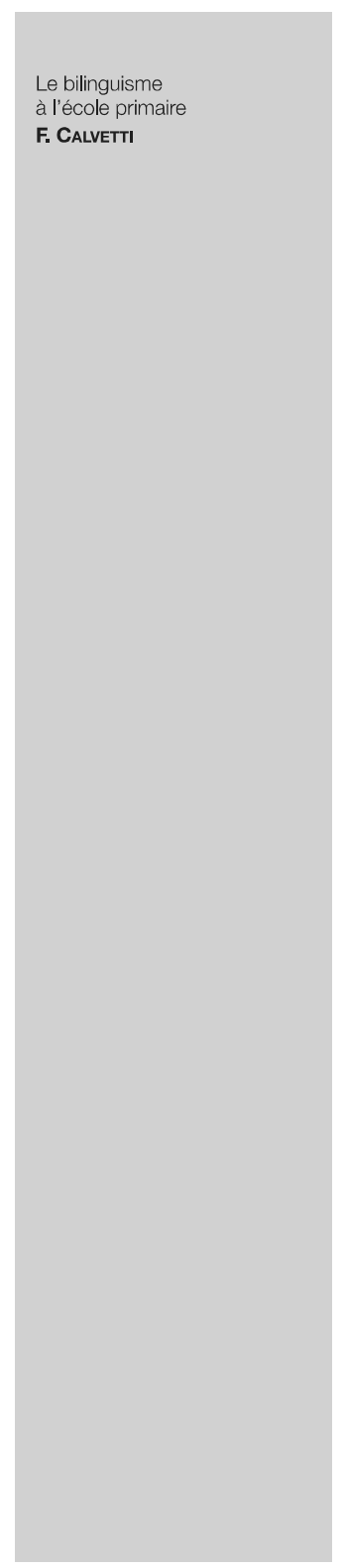

d'un code linguistique inconnu, sur le plan formatif pour ce qui concerne le rayonnement que peut donner l'approche d'une autre langue et d'une autre civilisation, sur le plan éthique car la connaissance des différences amène au respect pour la diversité.

* article paru dans Enfance 45, n 4, 1991, pp. 329-334. Republić avec autorisation. 